\title{
Préface : L'intégration du numérique dans les formations du supérieur
}

\section{Élisabeth Fichez et Alexandra Bal}

\section{(2) OpenEdition}

\section{Journals}

Édition électronique

URL : http://journals.openedition.org/edc/545

DOI : $10.4000 /$ edc.545

ISSN : 2101-0366

Éditeur

Université de Lille

Édition imprimée

Date de publication : 1 décembre 2007

Pagination : 7-15

ISBN : 978-2-9514961-8-7

ISSN : $1270-6841$

Référence électronique

Élisabeth Fichez et Alexandra Bal, « Préface : L'intégration du numérique dans les formations du supérieur », Études de communication [En ligne], Numéro spécial | 2007, mis en ligne le 21 janvier 2009, consulté le 21 septembre 2020. URL : http://journals.openedition.org/edc/545 ; DOI : https://doi.org/ 10.4000/edc. 545

Ce document a été généré automatiquement le 21 septembre 2020

(C) Tous droits réservés 


\title{
Préface : L'intégration du numérique dans les formations du supérieur
}

\author{
Élisabeth Fichez et Alexandra Bal
}

1 Ce numéro spécial renoue avec une thématique actualisée dans des numéros antérieurs de la revue Études de Communication, celle de la communication éducative médiatisée, interrogée à partir de l'hypothèse d'une industrialisation du champ de la formation ${ }^{1}$. Il est consacré aux analyses de chercheurs regroupés de 2003 à 2005 au sein d'une Équipe de Recherche en Technologie de l'éducation (ERTe) centrée sur les «Modèles économiques et enjeux organisationnels des campus numériques $»^{2}$. Autrement dit, il s'agissait de s'interroger sur les conditions de pérennisation de ces dispositifs en s'intéressant à deux d'entre elles, les conditions socio-économiques et les conditions socio-organisationnelles. Les terrains de référence privilégiés des articles sont donc, en France, les campus numériques mis en place sous forme de consortiums d'établissements au début des années 2000, et, au Canada, deux réalisations, le campus d'une université de l'Ontario (my.ryerson.ca) et une formation en ligne de la Teluq/Uqam «le DESS 'Santé mentale' $»^{3}$. Mais, le titre retenu pour le numéro indique que les préoccupations se sont élargies à une problématique plus générale, celle de l'intégration du numérique - pris au sens large de l'informatique, des réseaux et de la médiatisation - dans les formations $\mathrm{du}$ supérieur si bien que d'autres projets que ceux énoncés servent également de référents.

2 Si les chercheurs réunis dans cette étude ont des perspectives et des interprétations parfois divergentes, liées à leurs origines disciplinaires et à leur trajectoires personnelles ${ }^{4}$, ils sont tous d'accord cependant sur le fait que les campus numériques en question ne marquent qu'un moment particulier dans un temps d'innovation plus long, enclenché avant leur labellisation officielle par divers types de projets dont ils sont plus ou moins les héritiers et qui perdure à travers de nouveaux avatars, la politique ministérielle qui leur a donné naissance ayant elle-même connu un tournant dès 2002-2003 ${ }^{5}$. Après des monographies sur chacun des cas étudiés dans un premier 
temps, lesquelles ne pouvaient pas rendre compte de la complexité des évolutions qui se développent dans ce temps long, ce numéro a été conçu comme l'occasion de proposer un aperçu critique des projets qui pourra éventuellement servir de repère historique permettant d'appréhender les tendances de changements du système universitaire français à venir, les expériences canadiennes venant en contrepoint des tendances françaises. Cet aperçu critique ne se situe plus par conséquent comme dans les monographies à un niveau "méso", chacune d'entre elles portant sur une expérience particulière; les analyses prennent pour référence l'ensemble des campus (Fichez, Grevet) ou un sous-ensemble d'entre eux (Benchenna/Brulois, Bal/Combès) ou, lorsqu'ils en privilégient un en particulier (Moeglin, Deceuninck, Horn/Lamarche), il s'agit pour les auteurs d'approfondir une question de fond qui dépasse de loin l'entité en question et induit des mises en perspective avec d'autres contextes institutionnels (les campus numériques $v / s$ les campus traditionnels pour P. Moeglin; le campus Forse $v / s$ des formations à distance pour le Diplôme d'Accès aux Études Universitaires - DAEU - ou d'autres dispositifs de Formation à Distance en sciences de l'éducation pour J. Deceuninck, etc.).

3 Un premier ensemble de trois articles traite des prémices de ces projets et de leur devenir tel qu'analysé à partir des hypothèses qui ont fondé l'étude.

4 En ouverture, celui de Françoise Thibault, consacré à l'archéologie des campus en tant qu'initiative ministérielle, resitue le premier appel d'offres datant de l'année 2000 dans une lignée de discours annonçant dès les années 1990 une transformation radicale de l'existant (remplacement des universités classiques par des " universités virtuelles ») en lien avec la montée des réseaux. En fait l'auteur montre que le bon accueil fait au programme doit moins à la magie de la technique qu'à un climat politique très favorable et à des stratégies d'acteurs multiples. Mais elle insiste tout autant sur sa fragilité jamais démentie, en particulier dès lors qu'aux deux tiers du programme, en septembre 2001, un changement à la tête de la mission du supérieur a entraîné un virage dans la politique initiale. L'auteur conclut que le programme «Campus numérique ", malgré quelques réussites, correspond avant tout à une action publique avortée et constitue un exemple de plus des difficultés que rencontrent les dispositifs qui cherchent à faire reconnaître le métier d'enseignant dans l'université française.

5 L'article d'Elisabeth Fichez enchaîne sur une évaluation des réussites et échecs de ce programme, mesurés à l'aune de ses ambitions, à partir des mises en œuvre sur le terrain. Elle commence toutefois par s'interroger sur la qualification d'un projet comme "succès» ou "échec " en évoquant différentes démarches d'évaluation. Concevant les objets étudiés en tant que projets de dispositifs de formation médiatisés liés à des intentions innovantes, elle s'attache ensuite à repérer ces intentions - celles des politiques et celles des acteurs de terrain - en retient quatre principales et fait le point sur ce qu'il lui semble en être advenu en termes de tendances plus que de réalités stabilisées. Au final, il lui apparaît qu'il n'y a pas de réussite ou d'échec en soi ou global des campus, ces notions renvoyant au point de vue retenu et à la réalité sociale qu'il construit. Adoptant quant à elle le point de vue de l'analyste, elle rappelle qu'il s'agit ici de projets en incubation, pour lesquels l'appel ministériel a joué un rôle d'impulsion, et qu'en conséquence l'évaluation doit tenir compte de ce caractère évolutif et se faire non seulement en coupe transversale, mais aussi dans un temps plus long que celui de la politique publique qui les a portés. 
6 Patrice Grevet reprend quant à lui les hypothèses majeures posées dans le programme de recherche sur les modèles socioéconomiques d'enseignement supérieur repérables dans les trajectoires de campus numériques français. Partant d'une analyse empirique très attentive aux indications fournies par ces trajectoires, il franchit une étape de théorisation en proposant une lecture "néo-servicielle" de la numérisation de l'enseignement supérieur. La thèse s'inscrit chez lui aussi dans le long cours : après une phase initiale de numérisation pédagogique engagée il y a une dizaine d'années et non close à ce jour, celle-ci progresserait, de façon encore modeste, en s'inscrivant dans les cadres socioéconomiques familiers de l'enseignement supérieur. Bien évidemment ce processus n'a rien d'un long cours tranquille : s'y manifestent des contradictions, des indices, ou des amorces, signalant des éventualités de futurs changements plus profonds. Mais l'auteur insiste sur le fait que ceux-ci ne sont pas endogènes aux dispositifs, mais dépendent de transformations beaucoup plus générales liées aux tensions d'ensemble qui affectent l'enseignement supérieur, notamment en termes de gestion et de financement face aux conditions actuelles de la qualité.

7 De ce premier ensemble se dégage donc une première idée directrice : un processus de fond est engagé, celui de la numérisation pédagogique ${ }^{6}$, qui s'inscrit dans les pratiques familières au sein de l'enseignement supérieur sans pour autant que soit intervenue la transformation radicale prophétisée dans les années 90 .

8 Toutefois, les deux articles suivants, s'attachant à la question des «ressources virtuelles " rapportée à deux problématiques différentes, la figure d'« éditeur de ressources hors marché » et la logique en émergence du " courtage ", mettent l'accent plus sur les amorces de changements - voire, pour le premier, sur un modèle inédit en voie d'expansion - que sur les continuités, même si la prudence les amène eux aussi à mettre à jour obstacles et contradictions.

9 C'est l'analyse d'un campus atypique ${ }^{7}$ par rapport aux projets labellisés en 2000, l'Université Médicale Virtuelle Francophone, qui permet à François Horn et Thomas Lamarche de pointer la mise en place d'un modèle éditorial original, celui d'« éditeur hors marché ». Ils entendent à travers cette formule la création d'un portail permettant l'accès à des ressources, sans les produire directement, ce qui correspond à une fonction éditoriale transformée par la numérisation et Internet et dans laquelle le rôle de certification de ces ressources est essentiel. Ils insistent beaucoup sur un point qui leur apparaît déterminant dans les possibilités de généralisation de ce modèle indépendamment de son succès, mais aussi de ses limites - à savoir qu'il est fortement marqué par les spécificités de la profession médicale, notamment le monopole qu'exerce l'ordre médical dans la régulation du champ professionnel. Et pourtant, c'est bien ce modèle que le ministère a retenu en 2003, comme préfigurant les Universités Numériques Thématiques (UNT) attendues pour les autres disciplines. F. Horn et $\mathrm{T}$. Lamarche font l'hypothèse que l'absence d'un monopole professionnel comparable au sein de ces disciplines en limitera à tout le moins la puissance institutionnelle et posent par ailleurs la question de l'utilisation réelle des ressources éditées par des personnes autres que celles qui les ont développées.

10 C'est ce problème majeur qui structure la réflexion de Pierre Moeglin. C'est aussi un campus en particulier - C@mpusciences - qui inspire à l'auteur le développement d'une

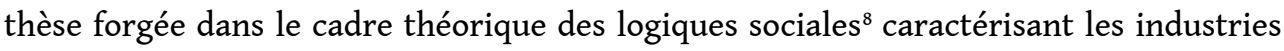
culturelles, celle du courtage, renvoyant à la fonction nécessaire d'un acteur capable de mettre en relation ressources et utilisateurs dans les campus numériques centrés sur 
des formations "dépaysées ", dont l'auteur s'attache à déceler, ne serait-ce que de manière idéal-typique, la spécificité par rapport aux campus classiques intégrant la numérisation dans les formations présentielles. C'est précisément, à son sens, parce que les acteurs de C@mpusciences ont refusé toute logique d'édition que se pose la question de l'utilité et de la viabilité des campus numériques, les ressources restant sous-employées dans leurs «trois vies" actuelles (la formation, l'auto-formation, l'autodidaxie). Mais la solution d'une "quatrième vie », ouverte par le courtage, reste toutefois partielle et d'une portée limitée, tant les obstacles institutionnels et organisationnels qui s'y opposent sont nombreux et importants.

11 Ces deux articles réinterrogent ainsi d'une façon différente ce que recouvre le vocable " campus numérique ", le modèle de l'édition hors marché et les UNT le tirant vers le portail de ressources en libre accès pour des usagers, sans souci de conformer une offre à une demande, le modèle de courtage le restreignant à des formations à distance marquées par une coupure producteurs/utilisateurs à laquelle il faut trouver un palliatif en adaptant a posteriori et sur mesure, des programmes, existants ou à créer, à des publics.

12 Un dernier ensemble de trois articles propose des éléments de bilan interrogeant les usages des dispositifs, analysant les tendances innovantes au croisement d'enjeux pédagogiques, industriels et institutionnels et discernant les processus dynamiques et les perspectives qu'ils laissent entrevoir.

13 Abdel Benchenna et Vincent Brulois soulignent la gageure que représente leur objectif, à savoir vouloir identifier des modes récurrents d'utilisation relativement intégrés dans la quotidienneté, alors que les campus labellisés (et continuant à fonctionner...) entrent à peine dans une phase opérationnelle. En même temps, s'il est une question particulièrement pertinente à observer au regard, en tout cas, de la politique ministérielle initiale, c'est bien celle de l'usage des dispositifs, clairement affichée comme critère de recevabilité des projets. Leur analyse va donc s'attacher à la compréhension des intentions de tout un ensemble d'acteurs à travers les discours et les actions accompagnateurs de l'opération et les premières réalisations dans deux campus (CampusCultura et C@mpusciences). Au final, ils confirment que, incontestablement, trouver des usagers et des usages est une entreprise difficile, plus sans doute que de produire des ressources! Et ils avancent l'idée que, si innovation il y a, elle est plus à chercher pour le moment dans ce que le projet de mise en place de campus numériques a engendré comme mouvement (coopérations entre acteurs, arbitrages réalisés sur certains points) que pour ce qu'il a produit comme ressources et dispositifs.

14 Alexandra Bal et Yolande Combès partent elles aussi de l'idée que les campus numériques marquent un moment particulier du temps long de l'innovation et considèrent les trois campus supports de leur analyse (C@mpuSciences et CampusCultura en France, my.ryerson.ca au Canada) comme une sorte d'observatoire expérimental à petite échelle d'évolutions plus générales. Elles lisent en effet les fluctuations de leurs orientations comme la recherche de consensus entre les impératifs pédagogiques et gestionnaires, ou encore comme la recherche de solutions pour débloquer des facteurs politiques ou organisationnels nécessaires à l'évolution des institutions. En France, elles notent ainsi que la différence d'importance attribuée à la relation et à l'information crée des tensions et donne lieu à des modes de fonctionnement organisationnels contrastés. En revanche, «my.ryerson.ca» offre 
l'exemple inédit d'un campus intégrateur qui agrège dans un système technologique centralisé les fonctions administratives, logistiques, d'enseignement et d'ingénierie. Constatant ainsi des tendances polymorphes empruntant à diverses formes industrielles, elles avancent l'idée que la résolution des problèmes passe sans doute par une modalité organisationnelle originale que l'on ne rencontre ni dans le champ de l'éducation et de la formation (sous ses formes traditionnelles), ni dans celui de l'édition... Ce qui nous ramène à la question des modèles, en émergence ou à venir...

Enfin, après avoir rappelé à son tour le caractère extrêmement mouvant des campus, Julien Deceuninck renvoie aux acteurs une série de réflexions et d'interrogations sur les dynamiques qui peuvent s'installer dans et au-delà de leur action. Se centrant sur des dispositifs de formation diplômants (Diplôme d'Accès aux Études Universitaires, Licence/master en sciences de l'éducation)il relève la coexistence de deux tendances complémentaires: des campus ( $c f$. Pégasus, Forse) qui se sont inscrits dans une dynamique numérique endogène propre au champ de la formation continue universitaire sans y faire disparaître la tendance à la concurrence entre établissements qui l'a emporté sur les velléités de mutualisation; des campus où se cherchent de nouveaux types d'équilibres d'ordre info-communicationnel dans les dispositifs de formation à travers l'oscillation entre la tentation de fixer des éléments communicationnels en documents (informationnels) et celle de faire passer des éléments informationnels de la forme de documents à celle d'échanges entre les participants (étudiants et enseignants). Il ne voit pas d'invention à proprement parler de nouvelles formes de communication pédagogique mais une réactualisation de la question centrale de l'industrialisation de la formation : celle de la part respective de l'enseignant et du document dans les dispositifs de formation et de leur possible réarticulation à travers les objets techniques.

Après les monographies publiées en ligne et des contributions dans un numéro récent de la revue Distances et Savoirs, ce numéro spécial d'Études de communication clôture ainsi l'exploitation des résultats de l'étude menée dans le cadre de l'ERTe ${ }^{10}$. Nous synthétiserons ainsi les trois traits importants qui nous semblent ressortir de la publication : elle constitue une sorte d'archive précieuse, à côté d'autres ${ }^{11}$, concernant une étape riche dans ce temps long qui s'impose comme seul cadre d'analyse pertinent des mutations dans le champ des formations du supérieur, tant du point de vue des pratiques des acteurs que de la modélisation des modes de fonctionnement qui le structurent ; s'agissant précisément de ces mutations, elle en analyse les indices et les tendances en croisant enquête de terrain minutieuse et énoncés théoriques, sans chercher à établir un consensus de surface entre hypothèses néo-servicielles et hypothèses liées aux logiques industrielles; enfin, elle renvoie une image modeste des évolutions en cours, sans pour autant les sous-estimer, en s'attachant bien sûr à la compréhension des dynamiques et modélisations endogènes aux dispositifs, mais aussi aux conditions exogènes beaucoup plus générales qui affectent les transformations de l'enseignement supérieur dans les pays développés. 


\section{NOTES}

1. On se reportera aux numéros 14 , «Éducation, formation: le temps de l'industrialisation?» (coord. par E. Fichez) en 1993 et 19, "Formation et espaces d'innovation» (coord. par E. Fichez et A. Payeur) en 1996.

2. Le MEN a assigné comme finalité à ce type d'équipes « la résolution des problèmes liés à l'éducation et la formation. Elles se justifient par une demande de praticiens de l'éducation et de la formation ou de responsables du système éducatif confrontés à un enjeu concret ou un problème ». Cf., Présentation des ERTE sur le site du gouvernement à l'adresse : http://www.education.gouv.fr/discours/2001/ dprost.htm\#2.

3. Ces campus étant très fréquemment cités dans les articles, nous avons fait figurer en annexe des fiches descriptives permettant au lecteur de disposer d'un certain nombre d'éléments factuels les concernant.

4. Nous renvoyons le lecteur à la consultation de la «Présentation " en ligne des études de cas qui explicite la nature des divergences dans les approches du fait des appartenances disciplinaires et des trajectoires différentes : «Le pari était que les tensions entre ces approches, au lieu d'être paralysantes, aboutiraient à des résultats fructueux dans leur confrontation avec les terrains. Il n'y avait pas l'illusion d'un empirisme juge de paix, ni l'intention d'arriver nécessairement à une problématique unifiée... Mais il y avait l'espoir que la dynamique des débats internes à l'ERTE et des confrontations aux terrains pousserait (au minimum) à affiner les problématiques, à mettre en lumière des données factuelles susceptibles d'ailleurs éventuellement d'être retournées contre les hypothèses ayant conduit à les dégager, à générer des éléments vraiment nouveaux de problématique... ». Cf. Résultats 2005 de l'ERTe (Équipe de Recherche Technologique éducation) "Modèles économiques et enjeux organisationnels des campus numériques", accessible à www.ifresi.univ-lille1.fr/SITE/ 2_Recherche/22_Programmes/ERTe/ERTe.htm ou à partir de la page d'accueil www.mshparisnord.org/.

5. Voir sur ce point à $F$. Thibault, «Autour des campus numériques français. Repères dans les initiatives du ministère en charge de l'enseignement supérieur", Distances et Savoirs, vol.4, $\mathrm{n}^{\circ} 1 / 2006$, p. 110.

6. Nous entendons par là à la fois la numérisation des ressources pédagogiques, le recours à des plates-formes logicielles pour la diffusion numérique des cours et de matériaux associés, la gestion des interactions synchrones ou asynchrones au travers d'outils configurés sur ces platesformes, etc.

7. Selon les auteurs, l'UMVF peut être considérée comme atypique pour deux raisons : alors que les campus numériques se sont constitués en rassemblant des équipes provenant de quelques universités partenaires, le projet de l'UMVF est de regrouper l'ensemble des facultés de médecine, ce qui s'est traduit par la création d'un Groupement d'Intérêt Public (GIP) ; par ailleurs son projet n'est pas le développement d'une offre de formation associée à un dispositif pédagogique mais la mise à disposition de ressources pédagogiques produites et utilisées par les universités partenaires.

8. Sur la question des logiques sociales, on se reportera à la fiche annexée "Logiques sociales ", in: G. Jacquinot-Delaunay et E. Fichez (éds.), «Université et TICE: chronique d'une innovation annoncée ", à paraître en 2008 chez De Boeck Université.

9. P. Moeglin, dans l'article ci-après, explicite ainsi le qualificatif : « La distance, séparation physique et décalage temporel, est structurellement inscrite dans le mode de fonctionnement du campus numérique ; grâce à elle, il s'agit de 'dépayser' la formation ».

10. Cf. Résultats 2005 de l'ERTE (ci-dessus note 5). Cf. aussi dans la revue Distances et Savoirs, vol. 4, $n^{\circ}$ 3/2006, E. Fichez : «Campus numériques : des ambitions à l'épreuve des terrains »; P. Grevet: 
«Une contradiction structurante dans la numérisation de l'enseignement supérieur »; A. Chaptal «États-Unis : le e-learning et le syndrôme d'Edison ».

11. Voir notamment l'ouvrage de G. Jacquinot-Delaunay et E. Fichez (2008, à paraître) cité dans la note 8 ci-dessus qui propose la chronique de l'expérience RUCA/UeL/C@mpusciences. Deux numéros (1 et 3) du volume 4 de la revue Distances et savoirs (2006) donnent un aperçu, sous le titre «Campus numériques universités virtuelles, et caetera " des réalités multiples recouvertes par le vocable en France et à l'étranger. Par ailleurs, toujours concernant la même période, on se reportera à l'ouvrage publié sous la direction de la Conférence des Recteurs des Universités italiennes «Les universités européennes à l'heure du e-learning: regards sur la Finlande, l'Italie et la France ", septembre 2006. Enfin, on consultera le numéro 169 (décembre 2006) de la revue Éducation Permanente consacré au thème «Pédagogie et numérique. Contradictions? Convergences?».

\section{AUTEURS}

\section{ÉLISABETH FICHEZ}

Elisabeth Fichez est professeur émérite à l'Université Lille 3, attachée au laboratoire GERIICO et membre du Séminaire Industrialisation de la Formation. Ses travaux de recherche portent sur des dispositifs de formation intégrant le numérique dans l'enseignement supérieur (campus numériques français et étrangers, pratiques collaboratives d'apprentissage par Internet, etc.). Outre la coordination de publications issues de ces deux terrains de recherche, elle co-dirige avec G. Jacquinot-Delaunay un ouvrage à paraître prochainement : «L'université et les TIC : chronique d'une innovation annoncée ». Adresse électronique : elisabeth.fichez-vallez@univ-lille3.fr.

\section{ALEXANDRA BAL}

Alexandra Bal est assistante/professeur de l'Université Ryerson et chercheure associée à la MSH - Paris Nord sur l'axe de recherche des industries de la culture et des arts. Ses travaux de recherche portent sur l'introduction des technologies de l'information et de la communication dans l'éducation universitaire de l'Ontario ainsi que sur le phénomène spécifique d'interdisciplinarité en jeu dans la création d'écologies médiatiques pédagogiques qui combinent les environnements virtuels et présentiels. Adresse électronique : abal@ryerson.ca. 